\title{
EQUIVALENT SPECTRAL MODEL FOR PEDESTRIAN-INDUCED FORCES ON FOOTBRIDGES: A GENERALIZED FORMULATION
}

\author{
Alberto Ferrarotti ${ }^{1}$ and Federica Tubino ${ }^{1}$ \\ ${ }^{1}$ DICCA, University of Genoa \\ Via Montallegro 1, 16145, Genova, Italy \\ alberto.ferrarotti@edu.unige.it; federica.tubino@unige.it
}

Keywords: Coherence Function, Dynamic Response, Footbridges, Pedestrian Dynamics, Serviceability Analysis, Spectral Model.

\begin{abstract}
This paper proposes a generalized equivalent spectral model of pedestrian-induced forces. The model can be applied for the serviceability analysis of footbridges subjected to unrestricted pedestrian traffic, but also in crowded conditions, in absence of pedestrianstructure interaction. It is based on the introduction of a suitable physically-based expression for the coherence function and a pedestrian density - velocity law. The proposed coherence model is a function of both pedestrian density and distance among pedestrians: it reduces to perfect uncorrelation for low pedestrian density (i.e. spatially unrestricted traffic), and to perfect correlation for high pedestrian density (i.e. crowded condition). Since the step frequency, the pedestrian speed and the pedestrian density are strictly correlated, the harmonic content of pedestrian-induced forces depends on pedestrian density. Based on the proposed equivalent spectral model of the loading, vibration serviceability analysis of footbridges subjected to any traffic conditions can be dealt with the classic methods of linear random dynamics. Similarly to classical procedures adopted in the wind engineering field, simple closed-form expressions for the evaluation of the maximum dynamic response are provided.
\end{abstract}




\section{INTRODUCTION}

Modern footbridges are very slender structures, often characterized by natural frequencies within the range of the dominant walking harmonics. As a consequence, they can be very sensitive to walking-induced vibration.

During its lifetime, a footbridge could be exposed to different loading scenarios, including a single crossing pedestrian, spatially unrestricted traffic, crowd traffic, joggers and runners and vandal loading; an exhaustive description can be found in [1]. The case of single crossing pedestrian can be modeled as a harmonic resonant moving load. The maximum structural response in such a case can be obtained in closed form [2]. Of course, the most common loading scenario is spatially unrestricted traffic. However, crowd loading can occur for footbridges placed in strategic areas or in particular conditions, such as during opening days.

In these last years, the vibration serviceability analysis of footbridges subjected to unrestricted pedestrian traffic has become a very topical subject in the international research panorama. A critical review of the different approaches for the estimate of the dynamic response in such a loading condition can be found in [3]. They may be classified into three categories: Monte Carlo simulations, simplified procedures ([4]-[7]) and spectral approach ([8], [9]). Monte Carlo simulations may be very time consuming and not practical from the usual design of footbridges. Simplified procedures have been proposed with the aim of simulating, in an approximate way, groups of pedestrians walking in a natural (i.e. random) way on a footbridge. The more widespread approach is based on the definition of equivalent uniform resonant loads producing the maximum dynamic response; this approach is usually very conservative, in particular for group loading. The spectral approach [9] is an interesting alternative: it consists in modelling the pedestrian-induced loading in unrestricted traffic conditions as a stationary random process through the analytical definition of a suitable spectral model.

On increasing pedestrian density, pedestrian traffic cannot be considered as unrestricted. The walking velocity monotonically decreases with growing density ([10]-[14]); furthermore, when pedestrian density is high enough, pedestrians are inclined to walk with the same step length [12]. Since walking velocity is directly proportional to step frequency and step length [15], the increase in pedestrian density produces a shift of the harmonic content of pedestrian-induced load towards lower values of frequency. It follows that some structures, with natural frequencies lower than those typical of unrestricted pedestrian traffic, may be affected by heavy vibrations if crowded conditions occur.

At the state of the art, to the authors' knowledge, the only attempt to estimate footbridges' dynamic response in crowded conditions relies on numerical simulations. The simulations are based on suitable models of pedestrian dynamics (e.g., [16]-[18]), representing the correlation among the forces exerted by each single pedestrian (e.g. [19]). These methods are very time consuming and are not appropriate for design purposes.

In this paper, a generalized equivalent spectral model is proposed, which can be applied for the serviceability analysis of footbridges also in crowded conditions in absence of pedestrianstructure interaction. Starting from the spectral model of the modal force proposed by Piccardo \& Tubino [9] and from the main rules and relationships of pedestrian dynamics, the generalized spectral model of pedestrian-induced forces is defined by introducing a suitable physically-based expression for the coherence function of the equivalent stationary random process and a pedestrian density - velocity law. The proposed coherence model is defined as a function of both pedestrian density and distance among pedestrians. It reduces to perfect uncorrelation for low pedestrian density (i.e. spatially unrestricted traffic), and to perfect correlation for high pedestrian density (i.e. crowded condition). Because of the fact that the step 
frequency, the walking speed and the pedestrian density are strictly correlated ([10]-[15] ), a dependency of the harmonic content of the force on the pedestrian density is introduced. Based on the proposed analytical generalized spectral model of the loading, simple expressions for the evaluation of the maximum dynamic response are sought for performing the vibration serviceability analysis of footbridges in a closed-form, similarly to classical procedures adopted in the wind engineering field. A numerical application to a real footbridge is finally proposed.

\section{EQUIVALENT SPECTRAL MODEL FOR SPATIALLY UNRESTRICTED TRAFFIC}

Unrestricted pedestrian traffic (i.e. pedestrians arriving in a random way and able to move undisturbed, each of them with his own characteristics in terms of loading amplitude, frequency, velocity and phase) was recently analyzed by Piccardo \& Tubino [9]. The authors proposed an equivalent spectral model of pedestrian-induced load, which allows to evaluate very easily the maximum structural response.

The force per unit length exerted by $N_{p}$ uncorrelated pedestrians can be expressed as the sum of the forces exerted by each single pedestrian, modeled as harmonic moving loads. Taking into account only the main harmonic for each pedestrian, the force per unit length can be written as follows [9]:

$$
f(x, t)=\sum_{i=1}^{N_{p}} \alpha_{i} G_{i} \sin \left(\Omega_{i}\left(t-\tau_{i}\right)+\Psi_{i}\right) \delta\left[x-c_{i}\left(t-\tau_{i}\right)\right]\left[H\left(t-\tau_{i}\right)-H\left(t-\tau_{i}-\frac{L}{c_{i}}\right)\right]
$$

where $\delta(\bullet)$ and $H(\bullet)$ are, respectively, the Dirac Delta function and the Heaviside step function, $t, x, L$ are, respectively, the time, the abscissa along the structure and the length of the structure. Furthermore $\alpha_{i}, G_{i}, \Omega_{i}, c_{i}, \tau_{i}$ are the dynamic load factor (DLF), the weight, the walking circular frequency, the walking velocity and the arrival time of the $i$-th pedestrian. All these quantities have to be probabilistically modeled. In particular, the step circular frequency $\Omega$, the pedestrian weight $G$, the DLF $\alpha$ and the walking velocity $c$ can be considered as Gaussian random variables. The phase-angle $\Psi$ among different pedestrians may be assumed as uniformly-distributed in the interval $[0,2 \pi]$. Considering pedestrian arrivals as Poisson events, then the time-lags between their arrivals are represented by an exponentiallydistributed random variable with an average return period $T=T_{p m} / N_{p}$, where $T_{p m}=L / c_{m}$ is the footbridge mean crossing time for a single pedestrian $\left(c_{m}\right.$ being the mean value of the walking speed).

By assuming linear behavior and classical damping, the equation of motion of the $j$-th principal coordinate $p_{j}$ can be expressed as:

$$
\ddot{p}_{j}(t)+2 \xi_{j} \omega_{j} \dot{p}_{j}(t)+\omega_{j}^{2} p_{j}(t)=\frac{1}{M_{j}} \int_{0}^{L} f(x, t) \varphi_{j}(x) d x
$$

where $\xi_{j} \omega_{j}$ and $M_{j}$ are, respectively, the $j$-th modal damping ratio, natural circular frequency and modal mass, and $\varphi_{j}$ the $j$-th mode shape.

Eq. (2) can be rewritten in non-dimensional form:

$$
\ddot{\tilde{p}}_{j}(\tilde{t})+2 \xi_{j} \dot{\tilde{p}}_{j}(\tilde{t})+\tilde{p}_{j}(\tilde{t})=\tilde{F}_{j}(\tilde{t})
$$

where the following non-dimensional quantities have been introduced: 


$$
\tilde{t}=\omega_{j} t \quad \tilde{p}_{j}=\frac{p_{j} M_{j} \omega_{j}^{2}}{\alpha_{m} G_{m}} \quad \tilde{f}(\tilde{x}, \tilde{t})=\frac{f(x, t) L}{\alpha_{m} G_{m}} \quad \tilde{x}=\frac{x}{L} \quad \tilde{F}_{j}(\tilde{t})=\int_{0}^{1} \tilde{f}(\tilde{x}, \tilde{t}) \varphi_{j}(\tilde{x}) d \tilde{x}
$$

being $\alpha_{m}$ the mean DLF, $G_{m}$ the mean pedestrian weight.

Furthermore, considering Eqs.(1), (2), (4), the non-dimensional modal force $\tilde{F}_{j}(\tilde{t})$ is given by:

$$
\tilde{F}_{j}(\tilde{t})=\sum_{i=1}^{N_{p}} \tilde{\alpha}_{i} \tilde{G}_{i} \sin \left(\tilde{\Omega}_{i}\left(\tilde{t}-\tilde{\tau}_{i}\right)+\Psi_{i}\right) \varphi_{j}\left(\tilde{\Omega}_{c i}\left(\tilde{t}-\tilde{\tau}_{i}\right)\right)\left[H\left(\tilde{t}-\tilde{\tau}_{i}\right)-H\left(\tilde{t}-\tilde{\tau}_{i}-\frac{1}{\tilde{\Omega}_{c i}}\right)\right]
$$

where the non-dimensional parameters are defined as:

$$
\tilde{\alpha}_{i}=\frac{\alpha_{i}}{\alpha_{m}} \quad \tilde{G}_{i}=\frac{G_{i}}{G_{m}} \quad \tilde{\Omega}_{i}=\frac{\Omega_{i}}{\omega_{j}} \quad \tilde{\Omega}_{c i}=\frac{c_{i}}{\omega_{j} L} \quad \tilde{\tau}_{i}=\omega_{j} \tau_{i}
$$

Let us define the mean non-dimensional walking speed $\tilde{\Omega}_{c m}$ analogously to $\tilde{\Omega}_{c i}$ in Eq. (6) with $c_{i}=c_{m}$. Assuming that the structural mode shape is sinusoidal, $\varphi_{j}(\tilde{x})=\sin (\pi \tilde{x})$, considering $\tilde{\alpha}_{i}=1, \tilde{G}_{i}=1, \tilde{\Omega}_{c i}=\tilde{\Omega}_{c m}, \tilde{\tau}_{i}=i / \tilde{\Omega}_{c m} N_{p}$ (i.e. focusing attention only on the randomness of the step frequency), and under the hypothesis that $\tilde{\Omega}_{c m}$ is very small compared with $\tilde{\Omega}_{i}$, the following expression for the power spectral density function (psfd) of the non-dimensional modal force is obtained [9]:

$$
S_{\tilde{F}_{j}}(\tilde{\Omega})=\frac{N_{p}}{4} p_{\tilde{\Omega}}(\tilde{\Omega})
$$

where $p_{\tilde{\Omega}}(\tilde{\Omega})$ is the probability density function of the non-dimensional circular step frequency $\tilde{\Omega}$.

By considering Eqs. (4), (6) and (7), the psdf of the $j$-th modal force can be rewritten in dimensional form as follows:

$$
S_{F_{j}}(\Omega)=\frac{N_{p}}{4} \cdot \frac{\left(\alpha_{m} G_{m}\right)^{2}}{\omega_{j}} p_{\Omega}(\Omega)
$$

The mean values of the DLFs and statistical moments of the step frequency can be found e.g. in Ref. [1], [9].

\section{PEDESTRIAN DYNAMICS}

When pedestrian density reaches values high enough, pedestrian traffic cannot be considered as unrestricted because pedestrians are inclined to synchronize their step length [12]. Furthermore, the harmonic content of the pedestrian-induced force may change because of the variation of both step frequency and velocity with pedestrian density ([10]-[15]). In order to model accurately these phenomena, it is necessary to study pedestrian dynamics.

To this day, a central problem in pedestrian dynamics is the relation between density and flow or velocity (fundamental diagram), which in general depends on the directionality of the flow (mono-directional, Section 3.1 or bi-directional, Section 3.2). 


\subsection{Mono-directional stream}

The mono-directional movement of pedestrians is the most widely studied scenario. All the proposed speed-density relations have some common features, concerning monotonic decreasing trend of the mean velocity $c_{m}$ with increasing density $\rho$ and the identification of some critical points [11]: the unrestricted flow speed $c_{u}$ (i.e. the mean maximum velocity), the critical density $\rho_{u}$ (i.e. the lower bound for unconstrained free walking), the jam density $\rho_{\max }$ (i.e. the maximum admissible density corresponding to null speed and flow). The free speed varies between about $1.1 \mathrm{~m} / \mathrm{s}$ and $1.5 \mathrm{~m} / \mathrm{s}$ [10], while the jam density may assume values up to 7 $\mathrm{Ped} / \mathrm{m}^{2}[10]$.

In the context of mono-directional flow, one of the most important laws is the fundamental diagram of Weidmann [13], a part of a review work in which the author summarized 25 different investigations. The author found a jam density $\rho_{\max }=5.4 \mathrm{Ped} / \mathrm{m}^{2}$ and a free flow speed probabilistically characterized as a Gaussian random variable with a mean value $c_{u m}=1.34 \mathrm{~m} / \mathrm{s}$ and $\sigma_{c u}=0.26 \mathrm{~m} / \mathrm{s}$ standard deviation. The mean velocity-density relation proposed by Weidmann, named "Kladek formula"[13], can be written in a generic form, as proposed by Venuti \& Bruno [11]:

$$
c_{m}(\rho)=c_{u}\left\{1-\exp \left[-\gamma \rho_{\max }\left(\frac{1}{\rho}-\frac{1}{\rho_{\max }}\right)\right]\right\}
$$

in which $\gamma$ depends on the travel purpose. By assuming $\rho_{\max }=5.4 \mathrm{Ped} / \mathrm{m}^{2}, c_{u}=c_{u m}=1.34 \mathrm{~m} / \mathrm{s}$ and $\gamma=0.354$, Eq. (9) matches with the original one proposed by Weidmann.

A significant explanation of the microscopic phenomena of the pedestrian traffic (i.e. interpedestrians relations) hidden behind the Kladek formula has been provided by [12]. The authors focused their attention on the changing in slope of the fundamental density - velocity law, and they identified different density domains in which the fundamental law can be considered about linear. The authors imputed these differences as a consequence of different effects due to microscopic characteristics of pedestrian traffic [12]. To support this claim, they performed an experimental campaign, in which flow characteristics were measured using both manual and automatic video procedures.

Starting from experimental results available in [12] and [15], Venuti et al. [11] proposed an interpretative model for the fundamental pedestrian diagram. The authors focused their attention on the Pedestrian Area Module (PAM), i.e. the surface required by a pedestrian during his motion. It should be noted that the PAM is the reciprocal of the pedestrian density. The PAM required by a pedestrian, with the hypothesis of rectangular surface, is $S=w d$ [11], being $w$ the lateral space and $d$ the forward distance. Both the quantities, in terms of mean values, can be considered as the sum of the dimension of a motionless pedestrian, and a dimension defined as function of the mean walking velocity thanks to the experimental results [11]:

$$
\left\{\begin{array}{l}
w_{m}\left(c_{m}\right)=w_{0}\left(1+0.62 \frac{c_{m}}{c_{u}}\right) \\
d_{m}\left(c_{m}\right)=d_{0}+1.06 c_{m}+b c_{m}^{10}
\end{array}\right.
$$

where $b=\left(2.08 c_{u}-d_{0}\right) / c_{u}^{10}, w_{0}$ and $d_{0}$ are the average lateral width and depth of the human body, which can be respectively assumed equal to $0.45 \mathrm{~m}$ and $0.36 \mathrm{~m}$ [11].

Moreover, the step frequency- speed relation, in terms of mean values, can be derived from a cubic fitting to the experimental data [11]: 


$$
n_{m}=0.35 c_{m}^{3}-1.59 c_{m}^{2}+2.93 c_{m}
$$

The mean value of the critical density $\rho_{u m}$ below which pedestrian traffic is unrestricted can be deduced from the definition of PAM, that could be intended as the reciprocal of the pedestrian density. By substituting $c_{m}=c_{u}=c_{u m}$ in Eq. (10), $\rho_{u m}=\left[d_{m} w_{m}\right]^{-1}=0.33 \mathrm{Ped} / \mathrm{m}^{2}$ is obtained. The value is very similar to the one suggested by Grundmann et al [27]. Then, by substituting $c_{m}=c_{u}$ into Eq. (11), the unconstrained step frequency $n_{u}$ can be obtained. It appears as Gaussian random variable with mean value $n_{u m}=1.91 \mathrm{~Hz}$ and $\sigma_{n u}=0.144 \mathrm{~Hz}$.

Figure 1 (a) shows the trend of the forward distance $d_{m}$ with the pedestrian mean speed. It should be noted that $d_{m}$ has linear trend with pedestrian velocity for speed values smaller than about $0.9 \mathrm{~m} / \mathrm{s}$. Let us indicate this value as lock-step speed, $c_{L S}$. Corresponding values in terms of pedestrian density and step frequency are, respectively, $\rho_{L S}=1.3 \mathrm{Ped} / \mathrm{m}^{2}$ (from Eq. (9)) and $n_{L S}=1.6 \mathrm{~Hz}$ (from Eq. (11)). According to Seyfried et al. [12], the marching in locksteps, which compensate the slower step frequency, are possible explanation for the linearity between the required length and the velocity.

Figure 1(b) shows the trend of the total outward distance with the pedestrian density. According to the same criteria used by Seyfried et al. [12], it is possible to individuate three domains in which the law $d_{m}-\rho_{m}$ is almost linear. In particular:

Domain I (unconstrained traffic). $\rho \leq \rho_{u m}$. The forward distance between pedestrians is constant and equal to its maximum value. Pedestrians' movement is completely free.

Domain II (correlated traffic). $\rho_{u m}<\rho \leq \rho_{L S}$. The forward distance between pedestrians quickly decreases with increasing in pedestrian density. In this domain, pedestrians' movement are not completely free.

Domain III (constrained traffic). $\rho>\rho_{L S}$. The forward distance between pedestrians is almost linear with pedestrian density, it is small and it slowly decreases with increasing in density. Marching in lock-steps totally occurred. Pedestrians' movements are completely constrained by the presence of other pedestrians.
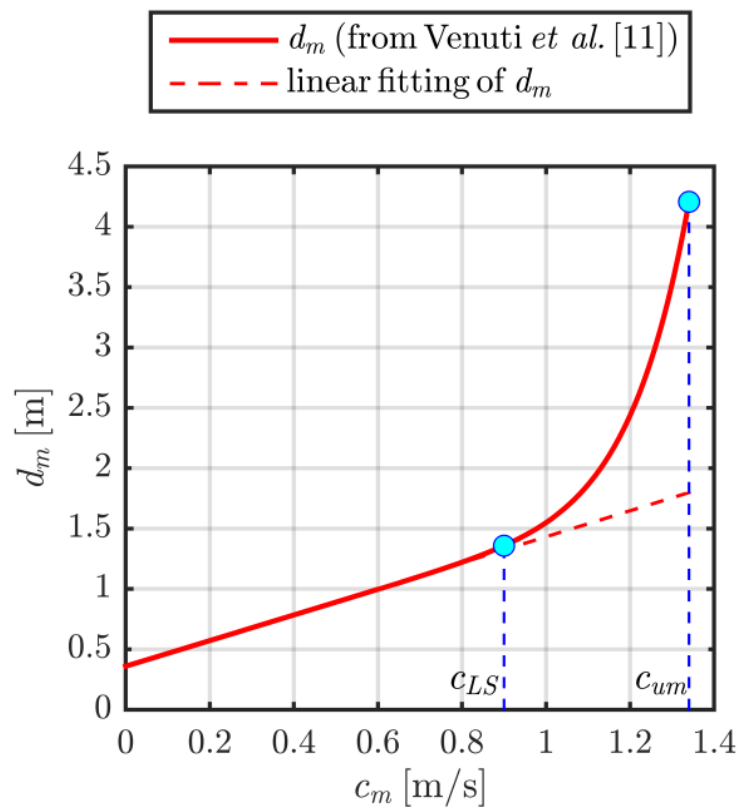

(a)

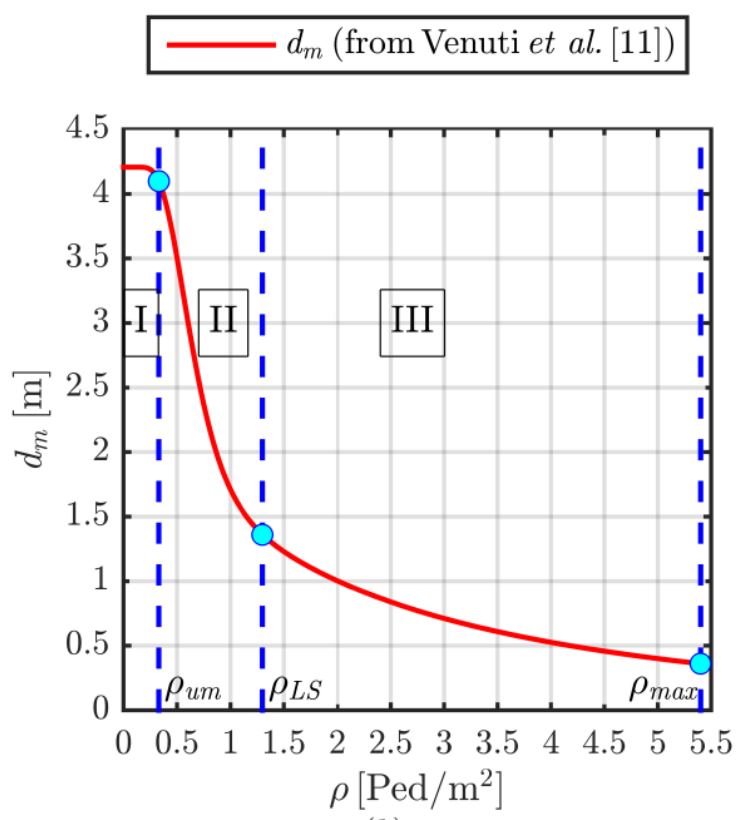

(b)

Figure 1: Trend of the mean forward distance with mean pedestrian velocity (a) and with pedestrian density (b) 


\subsection{Bi-directional stream}

In the last decades, problems related to bidirectional flows and its effects on pedestrian dynamics have gained increasing attention. First experimental studies about bi-directional streams were carried out by Older in 1968 [20] on footways in shopping streets. Several other experimental campaigns were carried out in transportation field [10]. At the same time, different microstructure methods for numerical simulation of pedestrian dynamics became an increasingly used instrument in order to study bi-directional streams (e.g. [16]-[18]).

Despite many experimental works in the transportation field and numerical works available in literature, a univocal proposal of a suitable fundamental law for bi-directional streams does not exist, to the authors' knowledge. Furthermore, at the state of art there is still no consensus whether the fundamental diagrams of mono- and bi-directional flows are different or not.

Fruin [22] states that the fundamental diagrams of mono- and bi-directional flow differ only slightly. Weidmann [13] neglected the differences in accordance with [22], but Navin and Wheeler [14] found a reduction of the flow in dependence of directional imbalances. Older [20] highlighted the phenomenon of lane formation as a natural system of limiting obstructions. Thanks to lanes, no differences occur between mono- and bi-directional streams, at least at low and medium densities [20].

A comprehensive description of the main phenomena that occur in bi-directional flows can be found Blue et al. [18]. They performed a large number of numerical simulations using CA Models, with the aim to study the fundamental diagram for the different types of bidirectional flows, over a range of directional splits in $10 \%$ increments from 100-0 (i.e. monodirectional flow) to 50-50 (i.e. balanced bi-directional flow). No significant differences among the mono-directional stream (i.e. 100-0) and bi-directional ones could be pointed out for stationary conditions.

Recently, Zhang et al. [21] carried out an experimental campaign with the aim to compare the fundamental diagrams of mono-directional flow with bi-directional one. They investigated bi-directional flows with balanced (50-50) and unbalanced (about 60-40) flow conditions. In 2015, Flötteröd and Lämmel [23], starting from experimental works of Zhang et al. [21], finally proposed a bi-directional pedestrian fundamental diagram, drawing inspiration from the cellular automata (CA) approach. At least until $\rho<2 \mathrm{Ped} / \mathrm{m}^{2}$, no meaningful mismatches can be found between the fundamental law proposed by Flötteröd and Lämmel [23] and the fundamental diagram for mono-directional streams (Eq. (9)).

\section{A PHISICALLY-BASED COHERENCE FUNCTION}

The coherence function of pedestrian-induced forces is a sparsely studied topic in the research panorama. Some studies on coherency, which concern relative phase angles among pedestrians, were done by Ebrahimpour et al. ([25], [26]). Piccardo \& Tubino [9], dealing with unrestricted pedestrian traffic, assumed as a coherence function the Dirac delta function in order to model perfect uncorrelation. At the state of art, a proper model of coherence function suitable for any pedestrian traffic conditions does not exist, to the authors' knowledge.

Some guidelines for a convenient definition of the coherence function were provided by Brownjohn et al. [8]. The spatial correlation in the pedestrian loading may be analogous to turbulent buffeting wind loads on a linear structure where cross-spectral density at two spatially separated points is assumed to diminish exponentially with increasing separation and frequency. For pedestrians, coherence depends on being able to see and keep up with others, and it is likely that it would drop to zero beyond a short separation distance [8]. 
A physically-based expression for the coherence function of the equivalent stationary random process is here introduced. It is composed by a unitary step function for small distance and an exponentially decreasing function for greater distance:

$$
\operatorname{Coh}_{f f}\left(x, x^{\prime}, \varepsilon, C\right)= \begin{cases}H\left(\frac{\left|x-x^{\prime}\right|}{\varepsilon}\right)-H\left(\frac{\left|x-x^{\prime}\right|}{\varepsilon}-1\right) & \text { if }\left|x-x^{\prime}\right| \leq \varepsilon \\ \exp \left[-C\left(\frac{\left|x-x^{\prime}\right|}{\varepsilon}-1\right)\right] & \text { otherwise }\end{cases}
$$

where $x$ and $x$ ' are the current abscissa, $\varepsilon$ is the width of the initial unitary step function and $C$ is the exponential decay coefficient. The main issue is the definition of the exponential decay coefficient $C$ and of the width of the initial step $\varepsilon$.

The width of the initial unitary step function $\varepsilon$ should be intended as a separation distance that each pedestrian interposes with others, in order to avoid contacts. The assumption of unitary step function is equivalent to assume that, on this distance, the single pedestrian has perfect synchronization with himself. It is possible to identify the separation distance between two pedestrians moving on a linear domain as strictly connected with the forward distance $d_{m}$. Moreover, by taking into account that, in the spectral model proposed at Section 2, each pedestrian is modeled as a moving harmonic punctual load (Eq. (1)), the separation distance between two pedestrians becomes of course $d_{m}-d_{0} / 2$.

The trend of $d_{m}$ with the pedestrian density is shown in Figure 1 (b). Considering Domain I, $\varepsilon$ should be identified as the forward distance corresponding to the mean free flow velocity $c_{u m}$. By substituting $c_{m}=c_{u m}$ in Eq. (10), $\varepsilon=d_{m}\left(c_{u m}\right)-d_{0} / 2 \cong 4 \mathrm{~m}$ is obtained.

The exponential part of the coherence function has the purpose to model the increase in synchronization between pedestrians that occurs when pedestrian density grows up (in Domains II and III). To do that, some limit behavior may be considered. For low pedestrian density (i.e. Domain I), pedestrians' walking is completely free [12] and the pedestrian-induced loading is uncorrelated. As a consequence, the coherence function may drop to zero very quickly, so the exponent must reach values high enough. On the contrary, for high pedestrian density (i.e. Domain III), marching in lock-step totally occurs [12]. Pedestrians' movements are completely constrained by others all around, and each of them is marching with the same step length and the same velocity [12]. Since the step length $l_{s}=c / n$ [11], then pedestrians are walking with the same step frequency. It follows that the pedestrian-induced loading is perfectly correlated. The coherence function may reach unitary values, so the exponent must drop to zero.

In order to satisfy all the conditions outlined above, it is reasonable to express the exponential decay coefficient as function of the mean step frequency. A suitable expression for the exponent can be identified through the following exponential function:

$$
C=C\left(n_{m}\right)=\exp \left(C_{1} n_{m}+C_{2}\right)
$$

where the two coefficients $C_{1}$ and $C_{2}$ can been obtained by enforcing the conditions $C\left(n_{u}\right)=C_{\max }=10, C\left(n_{L S}\right)=C_{\min }=0.1$. The limit values for the coefficient have been assumed based on numerical simulations: the exponential portion of the coherence function becomes negligible for values of the exponent greater than 10 , and it becomes almost unitary for exponents lower than 0.1. Under the hypothesis of $n_{u}=n_{u m}$, the values $C_{l}=22 \mathrm{~s}^{-1}$ and $C_{2}=-38$ are obtained. 
The exponent $C$ proposed in Eq. (13) could be rewritten as a function of the pedestrian density. In fact, the step frequency can be expressed as a function of the pedestrian density by substituting Eq. (9) into Eq.(11).

Figure 2 shows the trend of the exponential decay coefficient with respect to the step frequency (Figure 2 (a)) and pedestrian density (Figure 2 (b)).

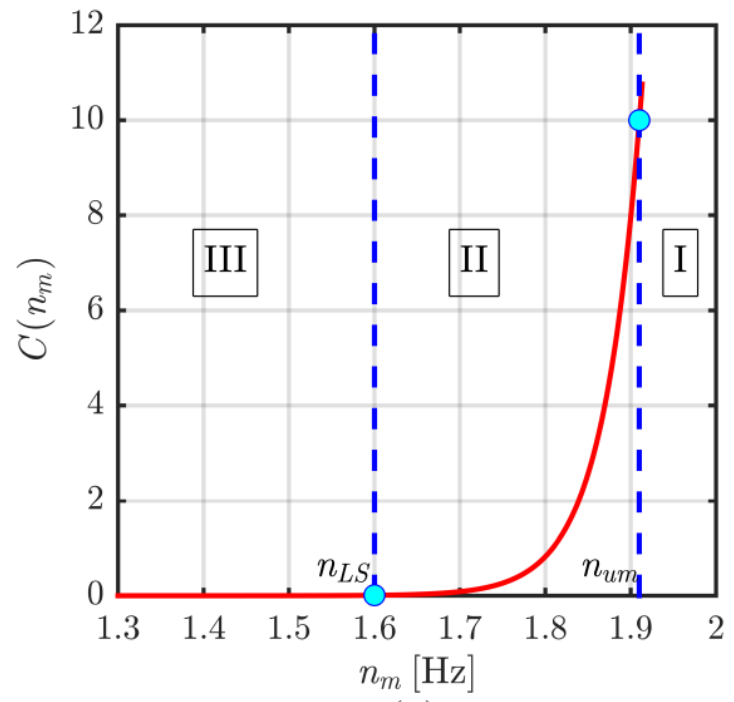

(a)

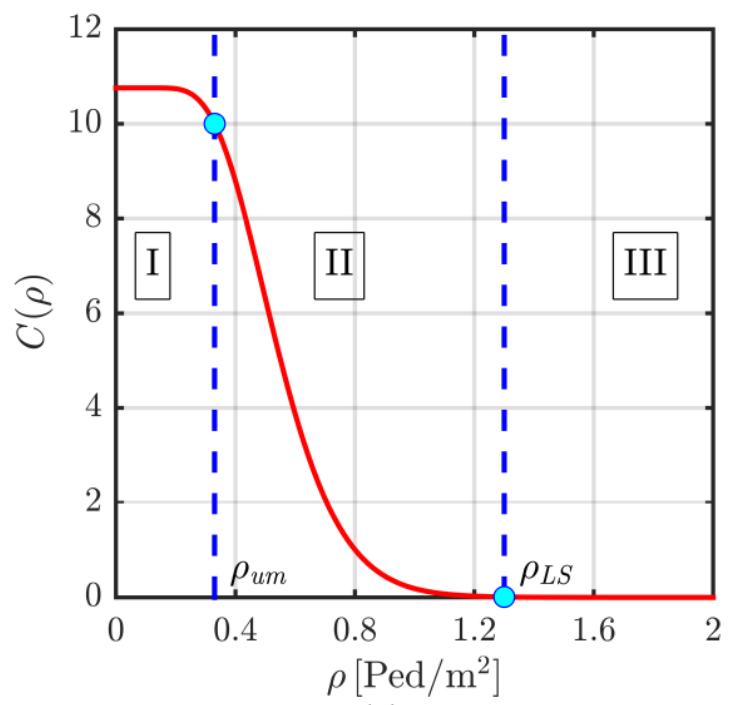

(b)

Figure 2: Exponential decay coefficient of the coherence function as a function of (a) mean step frequency and (b) pedestrian density

The proposed coherence function can be rewritten in the following non-dimensional form:

$$
\operatorname{Coh}_{\tilde{f f}}\left(\tilde{x}, \tilde{x}^{\prime}, \tilde{\varepsilon}, C\right)= \begin{cases}H\left(\frac{\left|\tilde{x}-\tilde{x}^{\prime}\right|}{\tilde{\varepsilon}}\right)-H\left(\frac{\left|\tilde{x}-\tilde{x}^{\prime}\right|}{\tilde{\varepsilon}}-1\right) & \text { if }\left|\tilde{x}-\tilde{x}^{\prime}\right| \leq \tilde{\varepsilon} \\ \exp \left[-C\left(\frac{\left|\tilde{x}-\tilde{x}^{\prime}\right|}{\tilde{\varepsilon}}-1\right)\right] & \text { otherwise }\end{cases}
$$

where $\tilde{\varepsilon}$ is the non-dimensional width of the initial unitary step function $\tilde{\varepsilon}=\varepsilon / L$. Figure 3 plots the resulting non-dimensional coherence function (Eq.(14)) as a function of the nondimensional distance, for fixed values of pedestrian density, assuming $\tilde{\varepsilon}=0.1$. As expected, the exponential portion is negligible for low values of the pedestrian density (i.e. $\rho<\rho_{\text {um }}$ ). Then, it monotonically increases with the pedestrian density and, finally, it reaches unitary value for $\rho>\rho_{L S}$. 


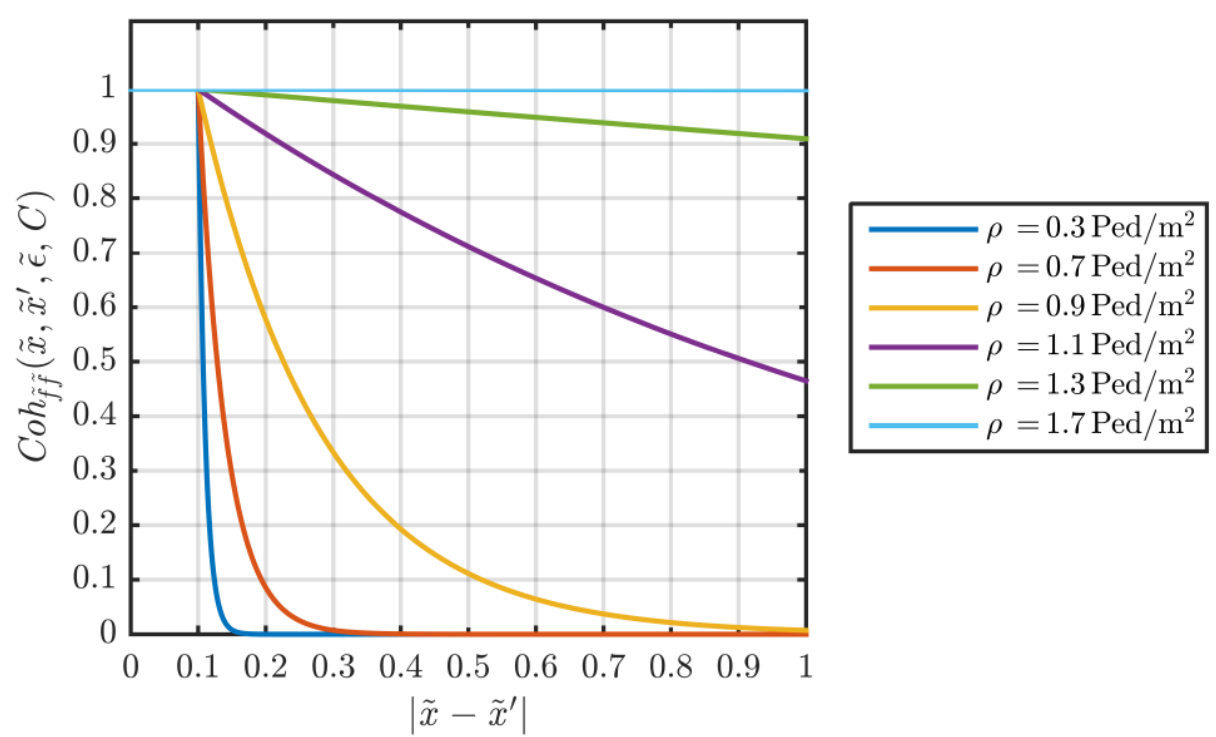

Figure 3: Proposed coherence function for different values of pedestrian density.

\section{THE GENERALIZED EQUIVALENT SPECTRAL MODEL OF THE HUMAN- INDUCED LOADING}

The power spectral density function of the non-dimensional modal force is given by:

$$
S_{F_{j}}(\tilde{\Omega})=\int_{0}^{1} \int_{0}^{1} S_{\tilde{f f}}\left(\tilde{x}, \tilde{x}^{\prime}, \tilde{\Omega}\right) \varphi_{j}(\tilde{x}) \varphi_{j}\left(\tilde{x}^{\prime}\right) \mathrm{d} \tilde{x} \mathrm{~d} \tilde{x}^{\prime}
$$

where $S_{\tilde{f f}}\left(\tilde{x}, \tilde{x}^{\prime} ; \tilde{\Omega}\right)$ is the cross-psdf of the non-dimensional force $\tilde{f}(\tilde{x}, \tilde{t})$. It's given by:

$$
S_{\tilde{f f}}\left(\tilde{x}, \tilde{x}^{\prime}, \tilde{\Omega}\right)=\sqrt{S_{\tilde{f}}(\tilde{x}, \tilde{\Omega}) S_{\tilde{f}}\left(\tilde{x}^{\prime}, \tilde{\Omega}\right)} \cdot \operatorname{Coh}_{\tilde{f f}}(\tilde{x}, \tilde{x}, \tilde{\varepsilon}, C)
$$

In Eq. (16) $S_{\tilde{f}}(\tilde{x}, \tilde{\Omega})$ is the psdf of the non-dimensional force and $\operatorname{Coh}_{\tilde{f}}(\tilde{x}, \tilde{x}, \tilde{\varepsilon}, C)$ is the coherence function described in Section 4. Under the assumption that the non-dimensional force has uniform psdf [9], $S_{\tilde{f}}(\tilde{x}, \tilde{\Omega})=S_{\tilde{f}}\left(\tilde{x}^{\prime}, \tilde{\Omega}\right)=S_{\tilde{f}}(\tilde{\Omega})$, considering Eqs. (15) and (16), the psdf of the non-dimensional force can be rewritten as follows:

$$
S_{F_{j}}(\tilde{\Omega})=S_{\tilde{f}}(\tilde{\Omega}) \cdot \chi_{j}(\tilde{\varepsilon}, C)
$$

where the admittance function $\chi_{j}(\tilde{\varepsilon}, C)$ has been introduced:

$$
\chi_{j}(\tilde{\varepsilon}, C)=\int_{0}^{1} \int_{0}^{1} \operatorname{Coh}_{\tilde{f f}}\left(\tilde{x}, \tilde{x} \tilde{x}^{\prime} ; \tilde{\varepsilon} ; C\right) \varphi_{j}(\tilde{x}) \varphi_{j}\left(\tilde{x}^{\prime}\right) \mathrm{d} \tilde{x} \mathrm{~d} \tilde{x}^{\prime}
$$

As outlined in Section 4, the exponential part of the coherence function is almost null under the hypothesis of unconstrained pedestrian traffic (i.e. $\rho<\rho_{u m}$ ). Moreover, it should be noted that the Eq. (14a) can be interpreted, from a perturbative point of view, as the incremental ratio of the Dirac delta function (i.e. the first derivative of the unitary step function), being the non-dimensional width of the initial unitary step function a small parameter. Thus, for $\rho<\rho_{u m}$ (i.e. in unrestricted traffic condition) the admittance function (Eq. (18)) assumes the following simplified expression: 


$$
\chi_{j}(\tilde{\varepsilon}, C) \cong \chi_{u j}(\tilde{\varepsilon}) \cong 2 \tilde{\varepsilon} \int_{0}^{1} \varphi_{j}(\tilde{x})^{2} \mathrm{~d} \tilde{x}
$$

Finally, assuming that the structural mode shape is sinusoidal [9], Eq. (19) becomes $\chi_{u j}(\tilde{\varepsilon}) \cong \tilde{\varepsilon}$.Considering Eqs. (7) and (17), the psdf of the non-dimensional equivalent distributed pedestrian-induced loading can be obtained:

$$
S_{\tilde{f}}(\tilde{\Omega})=\frac{N_{p}}{4 \tilde{\varepsilon}} p_{\tilde{\Omega}}(\tilde{\Omega})
$$

Eq. (20) can be rewritten in the dimensional form as follows:

$$
S_{f}(\Omega)=\frac{N_{p}}{4} \cdot \frac{\left(\alpha_{m} G_{m}\right)^{2}}{\omega_{j}} \cdot \frac{1}{\varepsilon L} \cdot p_{\Omega}(\Omega)
$$

The psdf of the modal force for a generic footbridge, with any mode shape and pedestrian density, can be evaluated through following steps: (i) assumption of the value of the free flow speed $c_{u}$ in the range $\left[c_{u m} \pm \sigma_{c u}\right.$ ], (ii) evaluation of the mean step frequency $n_{m}$ (Eqs. (9) and (11)) and of the exponential decay coefficient $C$ (Eq. (13)), (iii) computation of the admittance function $\chi_{j}(\tilde{\varepsilon}, C)$ (Eq. (18)) and (iv) application of Eq. (17) taking as mean value of the non-dimensional step frequency the one corresponding to the calculated mean step frequency $n_{m}\left(\tilde{\Omega}_{m}=2 \pi n_{m} / \omega_{j}\right)$.

\section{MAXIMUM STRUCTURAL RESPONSE}

Starting from the spectral model of the pedestrian-induced loading (Eq. (17)), the maximum dynamic response can be estimated through the classical methods of linear random dynamics [9]. The mean value of the maximum non-dimensional principal component $\ddot{\tilde{p}}_{j_{\max }}$ can be evaluated as follows [9]:

$$
\ddot{\tilde{p}}_{j_{\max }}=g_{\ddot{\tilde{p}}_{j}} \sigma_{\ddot{\tilde{p}}_{j}}
$$

where $\sigma_{\ddot{p}_{j}}$ is the standard deviation of $\ddot{\tilde{p}}_{j}$ and $g_{\ddot{\tilde{p}}_{j}}$ is the peak coefficient.

By assuming that the pedestrian-induced response is mainly resonant [9], the variance of $\ddot{\tilde{p}}_{j}$ can be estimated as [9]:

$$
\sigma_{\ddot{\tilde{p}}_{j}}^{2} \cong \frac{\pi}{4 \xi} S_{\tilde{F}_{j}}(1)
$$

being $S_{\tilde{F}_{j}}(1)$ the psfd of the non dimensional modal force, Eq. (17), calculated for $\tilde{\Omega}=1$.

The peak coefficient assumes the following expression [9]:

$$
g_{\ddot{p}_{j}}=\sqrt{2 \ln \left(2 v_{\ddot{p}_{j}}^{e} \tilde{T}\right)}+\frac{0.5772}{\sqrt{2 \ln \left(2 v_{\ddot{p}_{j}}^{e} \tilde{T}\right)}}
$$

in which the following non-dimensional parameters appear [9]: 


$$
\tilde{T}=\frac{N}{\tilde{\Omega}_{c m}} \quad v_{\ddot{\tilde{p}}_{j}}^{e}=\left(1.63 q_{\ddot{\tilde{p}}_{j}}^{0.45}-0.38\right) v_{\ddot{\tilde{p}}_{j}} \quad v_{\ddot{\tilde{p}}_{j}} \cong \tilde{n}_{j}=\frac{1}{2 \pi} \quad q_{\ddot{\tilde{p}}_{j}} \cong 2 \sqrt{\frac{\xi_{j}}{\pi}}
$$

being $N$ the number of consecutive pedestrian groups to be considered in order to satisfy the stationarity hypothesis. It should be noted that $\tilde{\Omega}_{c m}$ is the non-dimensional walking speed obtained by substituting in Eq. (6) the value $c_{i}=c_{m}(\rho)$ (Eq. (9)).

Starting from Eq. (22), the maximum acceleration of the principal coordinate $\ddot{p}_{j_{\max }}$ can be estimated as follows [9]:

$$
\ddot{p}_{j_{\max }}=\frac{\alpha_{m} G_{m}}{M_{j}} \ddot{\tilde{p}}_{j_{\max }}
$$

Then, assuming that the structural response is dominated by the $j$ th natural mode, the maximum acceleration in physical coordinates may be finally estimated as follows [9]:

$$
\ddot{q}_{\max }(x)=\varphi_{j}(x) \ddot{p}_{j_{\max }}
$$

\section{NUMERICAL APPLICATION.}

The proposed procedure is applied in order to estimate the maximum dynamic response of a real footbridge to various pedestrian traffic conditions, from completely unrestricted to fully-restricted pedestrian traffic. The structure is a slender footbridge in Milan, that was recently investigated through full-scale measurements [28]. The footbridge has a main span length $L=$ $60 \mathrm{~m}$. It's characterized by a first vertical flexural mode shape similar to the first mode of a double-hinge beam, with an identified natural frequency $n_{1}=2.17 \mathrm{~Hz}$, modal damping $\xi_{1}=$ 0.005 and modal mass $M_{l}=51000 \mathrm{~kg}$ [28].

The structural response can be estimated by the proposed formulation. It's assumed $G_{m}=$ $700 \mathrm{~N}$ and $\alpha_{m}=0.40$. According to [9], the standard deviation of the step frequency may be chosen $0.17 \mathrm{~Hz}$. In order to ensure stationarity $N=10$ is set.

By remembering that the free flow speed appearing in Eq. (9) is indeed a Gaussian random variable with mean value $c_{u m}=1.34 \mathrm{~m} / \mathrm{s}$ and standard deviation $\sigma_{c u}=0.26 \mathrm{~m} / \mathrm{s}$ [13] (i.e. mean unconstrained step frequency $n_{u m}=1.91 \mathrm{~Hz}$ and standard deviation $\sigma_{n u}=0.144 \mathrm{~Hz}$ ), the dynamic response of the footbridge may be evaluated by adopting a free flow speed in the range $\left[c_{u m} \pm \sigma_{c u}\right]$. Therefore different equivalent modal psdf may be obtained, and variable results may be found in terms of maximum structural response.

Figure 4 shows the maximum dynamic response of the bridge (Eq. (27) as a function of pedestrian density. The different curves correspond to different values of free-flow speed. 


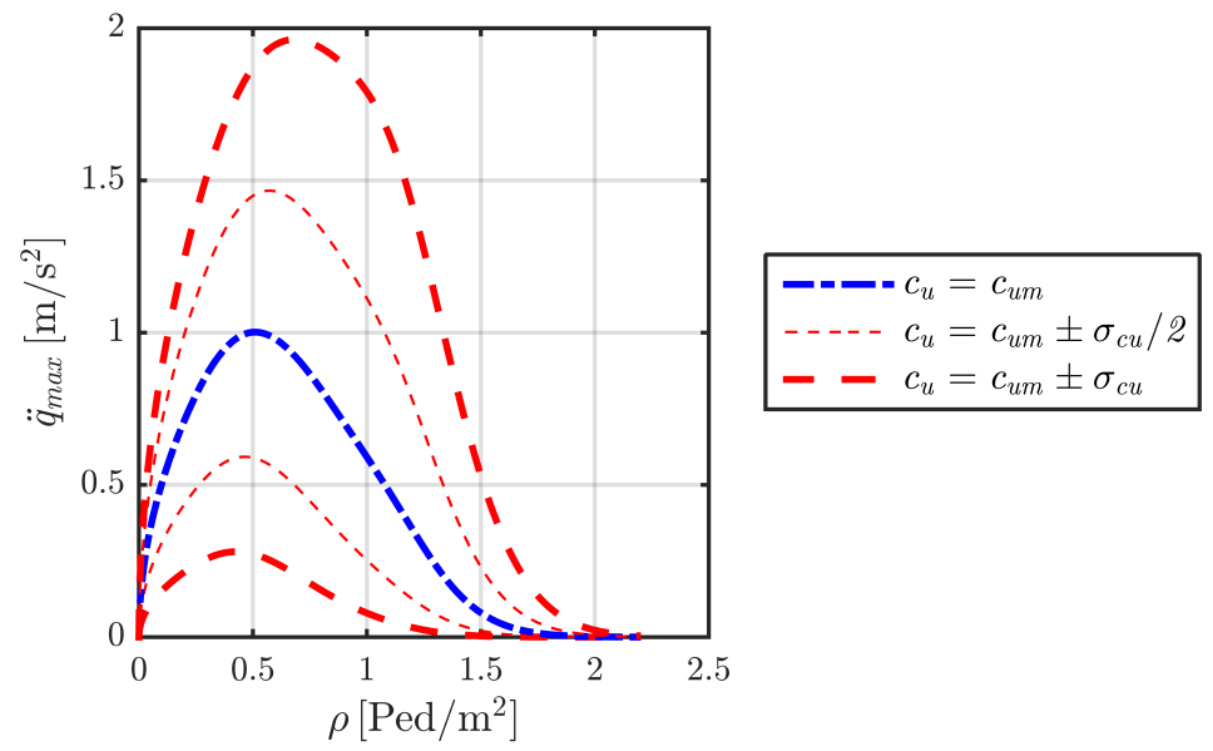

Figure 4: Maximum mid-span acceleration for a real footbridge for different values of free-flow speed

For low values of pedestrian density, the dynamic response tends to increase on increasing pedestrian density due to the growth of the number of pedestrians exciting the footbridge. Then, for high values of pedestrian density, the mean speed and mean step frequency progressively decrease; thus, the mean step frequency moves away from the structural frequency, and the structural response starts to decrease and progressively drops to zero, despite $N_{p}$ is still growing.

The analysis of the structural response shows that this real footbridge has a high sensitivity to human-induced vibrations. As a matter of fact, the structural response may reach values much higher than $1 \mathrm{~m} / \mathrm{s}$ (i.e. corresponding to minimum comfort, see [6] and [7]). Moreover, it demonstrates that small variations in terms of pedestrian velocity correspond to huge variations in terms of structural acceleration. This fact is due to the closeness between the first structural frequency and the mean step frequency. In order to avoid problems in terms of pedestrian comfort, the footbridge was recently equipped with tuned-mass dampers [29] .

\section{CONCLUSIONS AND PROSPECTS}

In this paper, the equivalent spectral model, originally introduced for unrestricted pedestrian traffic, has been generalized in order to account for correlation among pedestrians that can occur in crowded conditions. The generalization relies on the analysis of pedestrian dynamics and it is based on the introduction of a physically-based coherence function. The proposed spectral model allows to model both the change in the harmonic content of pedestrian-induced loading and the synchronization phenomenon on increasing pedestrian density.

A preliminary application to a real footbridge shows the potentiality of the model for the serviceability evaluation of footbridges in any traffic condition.

A validation (experimental and/or numerical) of the proposed model is necessary. The experimental validation could be provided by the comparison of the estimated maximum dynamic response with full-scale measurements in crowded conditions. The numerical validation may be carried out through numerical simulation of pedestrian flows based on suitable models of pedestrian dynamics. 


\section{REFERENCES}

[1] S. Zivanovic, A. Pavic, P. Reynolds. Vibration serviceability of footbridges under human-induced excitation: a literature review. Journal of Sound and Vibration, 279, 1-74, 2005 .

[2] G. Piccardo, F. Tubino, Dynamic response of Euler-Bernoulli beams to resonant harmonic moving loads. Structural Engineering and Mechanics, 44(5), 681-704, 2012.

[3] S. Zivanovic, A. Pavic, E.T. Ingolfsson. Modelling spatially unrestricted pedestrian traffic on footbridges. Journal or Structural Engineering ASCE, 136(10), 1296-1308, 2010.

[4] G. Piccardo, F. Tubino. Simplified procedures for the vibration serviceability analysis of footbridges subjected to realistic walking loads. Computers and Structures, 87, 890903, 2009.

[5] FIB, Guidelines for the Design of Footbridges, Bulletin 32. International Federation for Structural Concrete, Lauzanne, Switzerland, 2005

[6] SETRA, Footbridges - assessment of vibrational behaviour of footbridges under pedestrian loading. Technical Department for Transport, Roads and Bridges Engineering and Road Safety, Ministry of Transport and Infrastructures, Paris, France, 2006

[7] HIVOSS, Design of footbridges guidelines, Human induced vibrations of steel structures. $\quad$ http://www.stb.rwthaachen.de/projecte/2007/HIVOSS/download.phpRFS2-CT2007-00033 [22.04.09], 2008.

[8] J.M.W. Brownjohn, A. Pavic, P.A. Omentzetter. Spectral density approach for modelling continuous vertical forces on pedestrian structures due to walking. Canadian Journal of Civil Engineering, 31(1), 65-77, 2004.

[9] G. Piccardo, F. Tubino. Equivalent spectral model and maximum dynamic response for the serviceability analysis of footbridges. Engineering Structures, 40, 445-456, 2012.

[10] W. Daamen. Modelling passenger flows in public transportat facilities. PhD thesis, Delft University of technology, Department transport and planning, 2004

[11] F. Venuti, L. Bruno. An interpretative model of the pedestrian fundamental relation. C.R. Mecanique, 335, 194-200, 2007

[12] A. Seyfried, B. Steffen, W. Klingsch, M. Boltes. The fundamental Dyagram of Pedestrian Movement Revisite. Journal of Statistical Mechanics, 10, 2005

[13] U. Weidmann. Transporttechnik der Fußgänger. ETC Zürich, Ivt. Report no. 90, March 1993.

[14] P.D. Navin, R.J. Wheeler. Pedestrian Flow Characteristics. Traffic Engineering, 39(9), 30-33, 1969

[15] J.E. Bertram, A. Ruina. Multiple walking speed-frequency relations are predicted by constrained optimization. Journal of Theoretical Biology, 209, 445-453, 2001

[16] D. Helbing, P. Molnár. Social force model for pedestrian dynamics. Phisical Review E, 51(5), 4282-4286, 1995

[17] F. Dietrich, G. Köster. Gradient navigation model for pedestrian dynamics. Phisical Review E, 89(6), 2801-1-8, 2014 
[18] V.J. Blue, J.L. Adler. Cellular automata microsimulation for modeling bi-directional pedestrian walkway. Transportation Research Part B, 35, 293-312, 2001

[19] C.C. Caprani, J. Keogh, P. Archbold, P. Fanning. Characteristic Vertical Response of a Footbridge Due to Crowd Loading. 8th. International Conference on Structural Dynamics (EURODYN 2011), Leuven, Belgium, 2011

[20] S. Older. Movement of pedestrians on footways in shopping streets. Traffic Engineering and Control, 10(4), 160-163, 1968

[21] J. Zhang, W. Klingsch, A. Schadschneider, A. Seyfried. Ordering in bidirectional pedestrian flows and its influence on the fundamental diagram. Journal of Statistical Mechanics, 2, P02002, 2012

[22] J.J. Fruin. Pedestrian planning and design. Elevator World Inc., 1987.

[23] G. Flötteröd, G. Lämmel. Bidirectional pedestrian fundamental diagram. Transportation research Part B, 71, 194-212, 2015

[24] Transportation Research Board, Special Report 209, Highway Capacity Manual. National Research Council, Washington, DC, 1994.

[25] A. Ebrahimpour, L.L. Fitts. Measuring coherency of uman-induced rhythmic loads using force plates. ASCE Journal of Structural Engineering, 122, 829-831, 1996.

[26] A. Ebrahimpour, A. Hamam, R.L. Sack, W.N. Patten. Measuring and modeling dynamic loads imposed by moving crowds. ASCE Journal of Structural Engineering 122, 1468-1474, 1996

[27] H. Grundmann, M. Kreuzinger, M. Schneider. Dynamic Calculations of footbridges. Bauingenieur, 68, 215-225, 1993

[28] F. Tubino, L.Carassale, G. Piccardo, Human-induced vibrations on two lively footbridges in Milan, Journal of Bridge Engineering ASCE, tentatively accepted, 2015.

[29] F. Tubino, G. Piccardo, Tuned-Mass-Damper optimization for the mitigation of humaninduced vibrations of footbridges, Meccanica, 50(3), 809-824, 2015. 